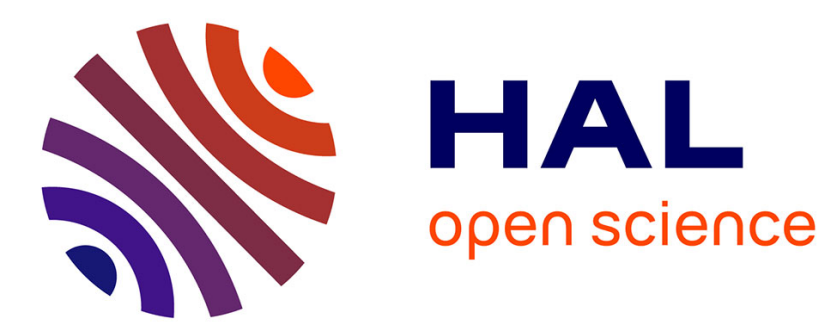

\title{
Derivation of magnetic inertial effects from the classical mechanics of a circular current loop
}

Stefano Giordano, Pierre-Michel Déjardin

\section{To cite this version:}

Stefano Giordano, Pierre-Michel Déjardin. Derivation of magnetic inertial effects from the classical mechanics of a circular current loop. Physical Review B, 2020, 102 (21), 10.1103/PhysRevB.102.214406 . hal-03278509

\author{
HAL Id: hal-03278509 \\ https://hal.science/hal-03278509
}

Submitted on 5 Jul 2021

HAL is a multi-disciplinary open access archive for the deposit and dissemination of scientific research documents, whether they are published or not. The documents may come from teaching and research institutions in France or abroad, or from public or private research centers.
L'archive ouverte pluridisciplinaire HAL, est destinée au dépôt et à la diffusion de documents scientifiques de niveau recherche, publiés ou non, émanant des établissements d'enseignement et de recherche français ou étrangers, des laboratoires publics ou privés. 


\title{
Derivation of magnetic inertial effects from the classical mechanics of a circular current loop
}

\author{
Stefano Giordano* \\ Institute of Electronics, Microelectronics and Nanotechnology - UMR 8520, Univ. Lille, \\ CNRS, Centrale Lille, ISEN, Univ. Valenciennes, LIA LICS/LEMAC, F-59000 Lille, France \\ Pierre-Michel Déjardin ${ }^{\dagger}$ \\ Laboratoire de Mathématiques et de Physiques, Université de Perpignan Via Domitia, 52 avenue Paul Alduy, F-66860 Perpignan, France
}

(Dated: November 19, 2020)

\begin{abstract}
The dynamical equation of a single magnetic moment constituted by a rigid circular current loop is derived from the mechanical Lagrange equations of motion, introducing the Lorentz force and the damping process, described by a well defined dissipative mechanism. It is demonstrated that magnetic inertial effects arise naturally by simple mechanical considerations and superimpose onto Gilbert original dynamical equation. The comparison with models proposed in the recent literature is drawn and discussed.
\end{abstract}

\section{INTRODUCTION}

The so-called Landau-Lifshitz-Gilbert (LLG) equation describes the orientational dynamics of a single magnetic dipole or the magnetization distribution in a given ferromagnetic material [1-3]. The first version of this equation was proposed by Landau and Lifshitz in 1935 to study the magnetic permeability dispersion in ferromagnetic bodies [4]

$$
\frac{d \vec{M}}{d t}=\gamma \vec{M} \wedge \vec{B}-\gamma \frac{\alpha}{M} \vec{M} \wedge(\vec{M} \wedge \vec{B})
$$

Here $\vec{M}$ is the dipole moment, $\gamma$ is the gyromagnetic ratio, $\alpha$ is the damping coefficient, and $\vec{B}$ is the effective magnetic induction. This equation has been modified in 1955 by Gilbert to describe the behavior of materials with large damping $[5,6]$

$$
\frac{d \vec{M}}{d t}=\frac{\gamma}{1+\alpha^{2}}\left[\vec{M} \wedge \vec{B}-\frac{\alpha}{M} \vec{M} \wedge(\vec{M} \wedge \vec{B})\right] .
$$

While the two equations exhibit the same mathematical form, they have a slightly different behavior, especially for large enough values of $\alpha$. However, this second-order difference can not be appreciated with experimental measurements and the choice between the models must be made on the basis of theoretical arguments [7-12]. The problem is still the subject of debate and different opinions can be found in the literature. For instance, Ref.[11] is in favor of the Gilbert version since it corresponds to an isotropic damping action, while Ref.[12] opts for the Landau-Lifshitz equation being coherent with irreversible thermodynamics.

Today, the LLG equation is very important since describes the magnetization dynamics in several systems and devices of crucial technological importance [13, 14]. A classical application concerns the modeling of the switching state in memory elements based on ferromagnetic materials $[15,16]$. More generally, magneto-electro-elastic structures [17-20], typically composed of piezoelectric and magnetelastic subsystems, are largely investigated being promising prototypes for

\footnotetext{
*stefano.giordano@univ-lille.fr

$\dagger$ dejardip@univ-perp.fr
}

the reduction of the energetic consumption in data storage and elaboration systems [21-23].

One of the most important feature of these systems is the stability of the stored information over long times. This means that thermal fluctuations must not alter the information recorded in the memory elements [24, 25]. To perform the analysis of this problem one can use the extension of the LLG equation with the additional term representing the thermal noise, as proposed by Brown in his pioneering works [26-29]. This generalization converts the LLG equation in a stochastic differential equation, or Langevin equation, which can be typically studied through the Fokker-Planck formalism [30, 31]. Several applications of this methodology can be found in the recent literature [32-36].

The theory of the magnetization dynamics is also of crucial importance to study the movement of a domain wall in ferromagnetic materials. The first important analytical result is given by the Walker solution describing, under simple assumptions, the one-dimensional steady state motion of a domain wall in a uniform magnetic field [37]. Because of the many advantages such as reliability, fast operation and low power consumption, devices based on domain walls are widely seen as promising tools for various applications, including data storage, sensing and logic [38-42]. Also in this context, the question of whether Landau-Lifshitz damping or Gilbert damping provides the more natural description of dissipative magnetization dynamics has been reopened [43]. It has been suggested that the Gilbert damping term is more adapted, showing the purely energy dissipative property also in the presence of nonconservative fields (e.g., with spintransfer torques) [44].

This state of affairs motivates the present readdressing of the magnetization dynamics. We propose here an original derivation of the corresponding equations based on the explicit consideration of the magnetic dipole structure as a charge distribution (first discrete and then continuous) rotating around a given axis with an intrinsic angular frequency (circular current loop). We firstly develop a model with a fixed angular velocity $\omega$ of charges. This hypothesis allows for the use of the Lagrange formalism for rheonomic systems, where the two-variables Lagrangian function explicitly depend on time (via the angular frequency $\omega$ ). Then, in order to obtain a more symmetric formalism, we introduce a second model with an 
arbitrarily varying angular velocity of the charges. In this case the system is scleronomous, but with three generalized coordinates. In both cases, while the dipole structure is invariant during the dynamics, the dipole orientation changes in response to the external actions. Within these approaches, the Lorentz and the damping forces can be naturally introduced without the need to specify a scalar magnetic potential and a Rayleigh dissipation function. This direct method allows therefore to obtain the explicit Lorentz contribution due to the dipole reorientation (always neglected) and a precise description of the damping force, defined by an explicit dissipation mechanism. Moreover, the introduction of the realistic magnetic dipole structure naturally generates in the final equations the inertial terms, corresponding of the second derivatives of the orientation angles with respect to the time. It is important to remark that these inertial terms and the Lorentz terms due to the reorientation are both of the order of $1 / \omega$ and therefore they are often neglected in practical applications. Nevertheless, the possible use of magnetic fields with extremely high frequencies (at pico- and femto-second time scales) has recently generated a wide interest for the generalization of the LLG equation with inertial effects [45-55]. In this context, our approach yields two generalized sets of second order differential equations for the magnetization dynamics, including the inertial effects and the Lorentz terms due to the reorientation. To get a simplified description, the concept of ideal dipole, corresponding to an infinitely small size, an infinitely large electric current, and a finite dipole moment, can be introduced through the limit $\omega \rightarrow \infty$. In this condition, our result reduces to a set of first order differential equations in perfect agreement with Eq.(2). This clearly explain why the LLG equation is a first order differential equation while the Lagrange equations are second order differential equations, coherently to the Newton law. Moreover, this analysis shows that the Gilbert form of the damping is more adapted than the Landau-Lifschitz form to describe dissipation in ferromagnetic materials.

It is interesting to underlying that, in constructing his equation, Gilbert himself reckoned that he could not conceive of a mechanical analogous system that undergoes his precessional equation, as the inertia tensor of such a system would have only one non-zero principal moment of inertia $[5,6]$. As discussed below, this point is clarified by the models proposed here, where three positive moments of inertia can be identified. This problem was noticed in particular by Wegrowe and co-workers who, in a long series of papers [45, 47-50], following previous experiments regarding ultrafast magnetization switching [56], proposed to complete the Gilbert equation by including inertial terms in their derivation. To this aim, they introduced a true inertial tensor and constructed a Lagrangian which kinetic energy is that of a symmetric top with one point fixed and the potential energy consists of the ferromagnetic one. They further utilized the Rayleigh dissipation function proposed by Gilbert $[5,6]$ and supported by Brown $[57,58]$ in order to account for damped precession. In combining these concepts with the gyromagnetic relation linking the angular momentum of the top and the magnetic moment, they were able to demonstrate that Eq.(2) is complemented by extra terms the importance of which rises in times scales which are shorter than that of gyromagnetic precession by orders of magnitude. At last, very recently, experimental evidence of such (resonant) inertial effects were achieved [55], and were found to occur at a probing frequency in the terahertz region, involving charateristic time scales that cannot be described by the classical Gilbert equation. Now, the derivation of Wegrowe et al. [47], although strongly indicating a close analogy between a magnetic dipole and a precessing and nutating symmetric top, does not allow to definitively conclude regarding this analogy, which is nevertheless extremely important for a qualitative understanding. Our analysis allows for a thorough explanation of this issue and better justifies the equation proposed by Wegrowe and co-workers.

To summarize, by meas of our approach, we clarify the following points concerning the dynamic equation for the magnetization reorientation:

1. we prove that a purely classical mechanics model is able to reproduce the LLG behavior with three positive moments of inertia;

2. this model supports the Gilbert damping term against the Landau-Lifschitz counterpart;

3. we show that the effect of the externally applied magnetic field can be directly described by the general Lorentz force, without the necessity to introduce a scalar magnetic potential;

4. we are able to precisely define the damping mechanism without resting on the Rayleigh function, which is convenient to use but hides the real dissipative process;

5. the proposed models automatically yield the terms describing the Lorentz force due to the reorientation of the magnetic dipole, a phenomenon always neglected;

6. importantly, the models here developed naturally lead to the inertial terms that must be added to the classical LLG equation to describe the specific response at picoand femto-second time scales;

7. in particular, the second model, when properly approximated, gives exactly the dynamic equation proposed by Wegrowe and co-workers [45, 47-50];

8. we provide evidence that the frequency response based on the two variants of our models and on the Wegrowe equation is exactly the same and show the classical ferromagnetic resonance together with the inertial or nutation resonance.

We believe that these points are important to give a clearer picture of the reorientation process of the magnetization vector and to get a better understanding of its underlying physics.

The paper is structured as follows. In Section II, we develop the model based on a circular current loop, where the electric charges rotate at constant angular velocity. Then, in Section III, we propose an alternative approach, with an arbitrarily varying angular velocity of the charges. This second approach shows a more elegant symmetry and allows to better explain the dynamic equation proposed by Wegrowe and 
a)

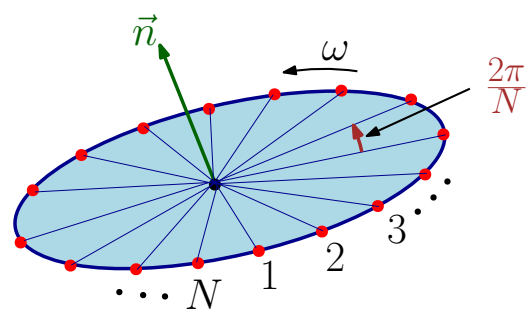

b)

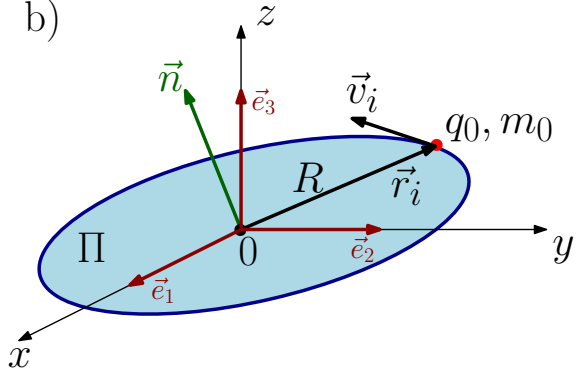

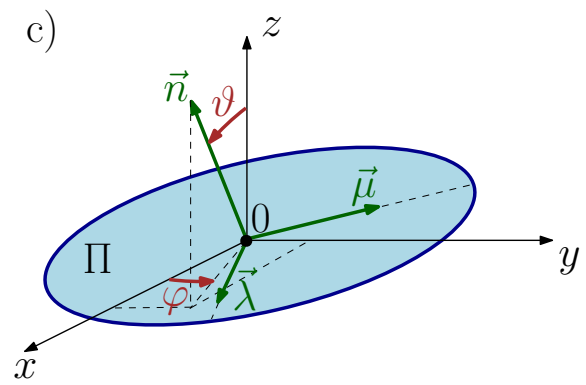

FIG. 1. Physical and geometrical description of the system. Panel a): discrete distribution of charge rotating around the center of the system with angular frequency $\omega$. Panel b): position and velocity vectors of the $i$-th particle with charge $q_{0}$ and mass $m_{0}$, and definition of the orthonormal bases $\left(\vec{e}_{1}, \vec{e}_{2}, \vec{e}_{3}\right)$. Panel c): definition of the precession and nutation angles $\varphi$ and $\vartheta$, and representation of the orthonormal bases $(\vec{\lambda}, \vec{\mu}, \vec{n})$ rigidly joined with the magnetic dipole .

co-workers. Finally, in Section IV we analyse the frequency response obtained through the proposed models and with the Wegrowe equation.

\section{DERIVATION OF GILBERT'S EQUATION FROM THE MOTION OF A CIRCULAR CURRENT LOOP WITH FIXED CENTER MODEL}

A magnetic dipole is typically considered as an elementary electric current flowing in a circular loop [59, 60]. Here, to follow this idea, we initially consider a very simple magnetic dipole structure characterized by a sequence of $N$ equally spaced material points (having mass $m_{0}$ and charge $q_{0}$ ) rotating on a circular trajectory of radius $R$ with angular frequency $\omega$. The angular spacing between the points is given by $2 \pi / N$ (see Fig.1a). The resulting dipole moment $M$ can be obtained through the classical expression $M=I S$, where $I=\frac{N q_{0} \omega}{2 \pi}=\frac{q \omega}{2 \pi}$ is the effective electric current in the loop, $q=N q_{0}$ is the total charge, and $S=\pi R^{2}$ is its surface. The dipole moment is therefore given by $M=\frac{1}{2} q \omega R^{2}$. Also, the total mass can be defined as $m=N m_{0}$. In order to obtain a continuous system for the magnetic dipole, we will analyze the limiting case with $N \rightarrow \infty, q_{0} \rightarrow 0$ and $m_{0} \rightarrow 0$ while keeping a finite value of $q$ and $m$. It is also important to define the classical gyromagnetic ratio $\gamma=\frac{q}{2 m}$, paying an important role in the dynamics of the dipole (according to the laws of classical physics, is the ratio of the magnetic moment to the angular momentum). We suppose that the discrete structure of the dipole is invariant (i.e., $q_{0}, m_{0}, N, R$, and $\omega$ are fixed in our process) whereas the dipole plane is subject to arbitrary reorientations around the geometrical center of the system. From the point of view of the analytical mechanics, this is a rheonomic system since the mechanical constraint implicates the time as an explicit variable [61]. It means that the material points have a preexisting orbital motion, independent on the dipole reorientation. It must be admitted that this is an operative assumption, which is difficult to be justified from an energetic point of view. Indeed, in our model, the origin of the power necessary to maintain the motion of the charges in the loop is not explained. Of course, the model we are going to present is useful to describe the dipole moment reorientation but is not able to discuss the origin of the spin behavior of magnetized matter. One of the difficulty comes from the fact that our model is based on classical physics whereas the origin of spin and of the magnetic dipole must be actually discussed within quantum physics. Indeed, the effective electric current in the loop corresponds, at the atomic scale, to electrons spin, nucleons spin and electrons orbital motions within the atom structure, and all these phenomena can be only explained through quantum mechanics. Therefore, the energetics of spins or dipoles is beyond the scope of the present paper.

Anyway, to better explain the system geometry, we observe that the plane where the dipole is confined can be characterized by its unit normal vector $\vec{n}$

$$
\vec{n}=(\cos \varphi \sin \vartheta, \sin \varphi \sin \vartheta, \cos \vartheta)
$$

where $\varphi$ and $\vartheta$ are the precession and nutation angles, respectively (see Fig.1b and 1c). Our aim is to find the equations governing the time evolution of $\varphi$ and $\vartheta$ based on the external actions applied to the magnetic dipole. On the moving plane of the dipole, we can define a couple of unit vectors such that they coincide with $\vec{e}_{1}$ and $\vec{e}_{2}$ when $\varphi=\vartheta=0$. We have

$$
\begin{aligned}
& \vec{\lambda}=\frac{\partial \vec{n}}{\partial \vartheta}=(\cos \varphi \cos \vartheta, \sin \varphi \cos \vartheta,-\sin \vartheta) \\
& \vec{\mu}=\frac{1}{\sin \vartheta} \frac{\partial \vec{n}}{\partial \varphi}=(-\sin \varphi, \cos \varphi, 0) .
\end{aligned}
$$

Therefore, $(\vec{\lambda}, \vec{\mu}, \vec{n})$ represents an orthonormal bases rigidly bound to the loop (see Fig.1c). Now, we can introduce the motion $\vec{r}_{i}(t)$ of the $i$-th point charge $q_{0}$ as follows

$$
\vec{r}_{i}(t)=\vec{\lambda} R \cos \left(\omega t+p_{i}\right)+\vec{\mu} R \sin \left(\omega t+p_{i}\right),
$$

which manifestly shows the rheonomic character of the constraint since the time is explicit within the terms $\cos \left(\omega t+p_{i}\right)$ and $\sin \left(\omega t+p_{i}\right)$. Here, $p_{i}=\frac{2 \pi}{N}(i-1)$ with $i=1, \ldots, N$. Of course, from the trajectory $\vec{r}_{i}(t)$, we can also define the velocity vector $\vec{v}_{i}(t)=\frac{d \vec{r}_{i}}{d t}$ (see Fig.1b). Within the Lagrangian mechanics, $\varphi(t)$ and $\vartheta(t)$ assume the role of generalized coordinates. By adopting this formalism, the motion equations 
can be written as [61]

$$
\begin{aligned}
\frac{d}{d t} \frac{\partial T}{\partial \dot{\vartheta}}-\frac{\partial T}{\partial \vartheta} & =Q_{\vartheta} \text { with } Q_{\vartheta}=\sum_{i=1}^{N} \vec{F}_{i} \cdot \frac{\partial \vec{r}_{i}}{\partial \vartheta}, \\
\frac{d}{d t} \frac{\partial T}{\partial \dot{\varphi}}-\frac{\partial T}{\partial \varphi}=Q_{\varphi} \text { with } Q_{\varphi} & =\sum_{i=1}^{N} \vec{F}_{i} \cdot \frac{\partial \vec{r}_{i}}{\partial \varphi},
\end{aligned}
$$

where $T=\frac{1}{2} m_{0} \sum_{i=1}^{N} \vec{v}_{i} \cdot \vec{v}_{i}$ is the kinetic energy of the particles system, $\vec{F}_{i}$ is the total force applied to the $i$-th particle, and $\left(Q_{\vartheta}, Q_{\varphi}\right)$ are the so-called generalized forces [61]. We discuss below the physical contributions to $\vec{F}_{i}$. We remark that Eqs.(7) and (8) represent the most general formulation of Lagrangian mechanics where both conservative and dissipative actions can be envisaged [61]. Now, to obtain the kinetic energy, we develop the velocity vectors as follows

$$
\begin{aligned}
\vec{v}_{i}(t)= & \dot{\vec{\lambda}} R \cos \left(\omega t+p_{i}\right)-\vec{\lambda} \omega R \sin \left(\omega t+p_{i}\right) \\
& +\dot{\vec{\mu}} R \sin \left(\omega t+p_{i}\right)+\vec{\mu} \omega R \cos \left(\omega t+p_{i}\right) .
\end{aligned}
$$

From the orthonormality properties $\vec{\lambda} \cdot \vec{\mu}=0, \vec{\lambda} \cdot \vec{\lambda}=1, \vec{\mu}$. $\vec{\mu}=1$, we get by differentiation $\dot{\vec{\lambda}} \cdot \vec{\mu}+\vec{\lambda} \cdot \dot{\vec{\mu}}=0, \dot{\vec{\lambda}} \cdot \vec{\lambda}=0$, and $\dot{\vec{\mu}} \cdot \vec{\mu}=0$. Hence, we simply obtain

$$
\begin{aligned}
\vec{v}_{i}(t) \cdot \vec{v}_{i}(t)= & \dot{\vec{\lambda}} \cdot \dot{\vec{\lambda}} R^{2} \cos ^{2}\left(\omega t+p_{i}\right)+\dot{\vec{\mu}} \cdot \dot{\vec{\mu}} R^{2} \sin ^{2}\left(\omega t+p_{i}\right) \\
& +\omega^{2} R^{2}+2 \dot{\vec{\lambda}} \cdot \dot{\vec{\mu}} R^{2} \cos \left(\omega t+p_{i}\right) \sin \left(\omega t+p_{i}\right) \\
& -2 \vec{\lambda} \cdot \dot{\vec{\mu}} \omega R^{2} .
\end{aligned}
$$

To further simplify this expression we can use the relationships

$$
\begin{aligned}
& \dot{\vec{\lambda}} \cdot \dot{\vec{\lambda}}=\dot{\vartheta}^{2}+\dot{\varphi}^{2} \cos ^{2} \vartheta, \\
& \dot{\vec{\mu}} \cdot \dot{\vec{\mu}}=\dot{\varphi}^{2}, \\
& \vec{\lambda} \cdot \dot{\vec{\mu}}=-\dot{\varphi} \cos \vartheta, \\
& \dot{\vec{\lambda}} \cdot \dot{\vec{\mu}}=\dot{\varphi} \dot{\vartheta} \sin \vartheta .
\end{aligned}
$$

We eventually get the kinetic energy in the final form

$$
\begin{aligned}
T= & \frac{1}{2} m_{0} R^{2} \sum_{i=1}^{N}\left[\dot{\vartheta}^{2} \cos ^{2}\left(\omega t+p_{i}\right)+\dot{\varphi}^{2}+\omega^{2}\right. \\
& -\dot{\varphi}^{2} \sin ^{2} \vartheta \cos ^{2}\left(\omega t+p_{i}\right)+2 \omega \dot{\varphi} \cos \vartheta \\
& \left.+2 \dot{\varphi} \dot{\vartheta} \sin \vartheta \cos \left(\omega t+p_{i}\right) \sin \left(\omega t+p_{i}\right)\right] .
\end{aligned}
$$

Now, we can apply the continuous limit to this result. To do this, we use the relation $m_{0}=m / N$ and we transform the sum into an integral over $p$ (i.e. over the distribution of charge and mass) by observing that $d p \simeq 2 \pi / N$. Hence, we get

$$
\begin{aligned}
T= & \frac{1}{2} m R^{2} \frac{1}{2 \pi} \int_{0}^{2 \pi}\left[\dot{\vartheta}^{2} \cos ^{2}(\omega t+p)+\dot{\varphi}^{2}+\omega^{2}\right. \\
& -\dot{\varphi}^{2} \sin ^{2} \vartheta \cos ^{2}(\omega t+p)+2 \omega \dot{\varphi} \cos \vartheta \\
& +2 \dot{\varphi} \dot{\vartheta} \sin \vartheta \cos (\omega t+p) \sin (\omega t+p)] d p .
\end{aligned}
$$

A straightforward integration delivers

$$
\begin{aligned}
T & =\frac{1}{2} m R^{2}\left(\frac{1}{2} \dot{\vartheta}^{2}+\dot{\varphi}^{2}+\omega^{2}-\frac{1}{2} \dot{\varphi}^{2} \sin ^{2} \vartheta+2 \omega \dot{\varphi} \cos \vartheta\right) \\
& =\frac{1}{4} m R^{2}\left(\dot{\vartheta}^{2}+\dot{\varphi}^{2} \sin ^{2} \vartheta\right)+\frac{1}{2} m R^{2}(\omega+\dot{\varphi} \cos \vartheta)^{2}
\end{aligned}
$$

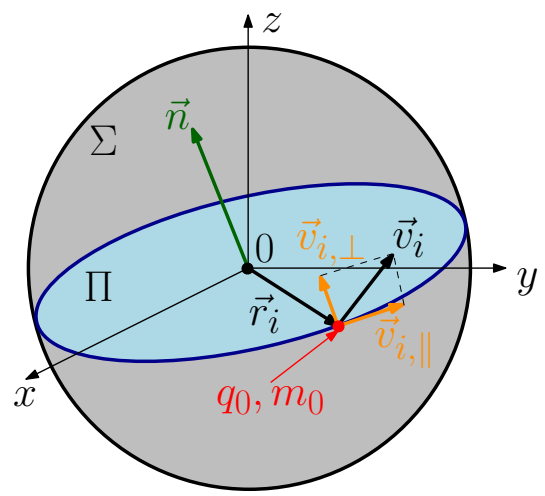

FIG. 2. Instantaneous decomposition of the charge velocity $\vec{v}_{i}$ in the two components tangent $\vec{v}_{i, \|}$ and perpendicular $\vec{v}_{i, \perp}$ to the instantaneous position of the dipole circle. The perpendicular component is crucial for the introduction of the damping force. While $\Pi$ represents the dipole plane, $\Sigma$ is the sphere circumscribed to the dipole.

This expression allows us to calculate the left hand side of the Lagrange equations for our system as follows

$\frac{d}{d t} \frac{\partial T}{\partial \dot{\vartheta}}-\frac{\partial T}{\partial \vartheta}$

$=m \omega R^{2}\left[\dot{\varphi} \sin \vartheta+\frac{1}{2 \omega}\left(\ddot{\vartheta}+\dot{\varphi}^{2} \sin \vartheta \cos \vartheta\right)\right]$,

$\frac{d}{d t} \frac{\partial T}{\partial \dot{\varphi}}-\frac{\partial T}{\partial \varphi}$

$=m \omega R^{2}\left[-\dot{\vartheta} \sin \vartheta+\frac{1}{2 \omega}\left(2 \ddot{\varphi}-\ddot{\varphi} \sin ^{2} \vartheta-2 \dot{\vartheta} \dot{\varphi} \sin \vartheta \cos \vartheta\right)\right]$,

where we separated the terms proportional to $\omega$ from the others. We will discuss this separation below. In order to complete the Lagrange equations of motion, we need to elaborate the generalized forces $Q_{\vartheta}$ and $Q_{\varphi}$. To do this, we assume that the charged particles are subjected to two kinds of forces.

Firstly, we consider the Lorentz force $\vec{F}_{i, L}=q_{0} \vec{v}_{i} \wedge \vec{B}$ generated by an external magnetic induction $\vec{B}=\left(B_{x}, B_{y}, B_{z}\right)$. Of course, this is an effective magnetic induction representing many real factors: the Zeeman effect induced by an applied magnetic field, the demagnetization effect generated by the magnetic field created by the magnetization itself, the exchange effect depending of the gradients of the magnetization, the magnetic anisotropy effect induced by the crystalline structure of the materials, and the magnetoelastic effects generated by the interaction of magnetic and elastic fields. Typically, all these contributions are summed up through an effective energy function, which can be derived with respect to the magnetization to give the effective magnetic induction $\vec{B}$ applied to the dipole [57, 58].

Secondly, we introduce a damping force describing the effective viscous drag acting opposite to the reorientation motion of the dipole plane $\Pi$. We precisely describe the dissipation mechanism as follows. At a given time $t$, we have a given orientation of $\Pi$ identified by $\vec{n}$ or, equivalently, by $\varphi$ and $\vartheta$ (see Fig.2). At that time $t$, each charge velocity $\vec{v}_{i}$ can be decomposed in the two components tangent, $\vec{v}_{i, \|}$, and perpendicular, $\vec{v}_{i, \perp}$, to the instantaneous position of the dipole 
circle (see Fig.2). Indeed, while each particle velocity is tangent to the sphere $\Sigma$ circumscribed to the dipole, it is not tangent to the dipole circle because it is in motion $(\dot{\vartheta} \neq 0$ and $\dot{\varphi} \neq 0$ ). Now, since we want to describe the damping of the dipole motion and not of the particle motion, we apply a drag force opposite to the perpendicular component of each instantaneous particle velocity. Hence, we define the damping force $\vec{F}_{i, D}=-k_{0} \vec{v}_{i, \perp}=-k_{0}\left(\vec{v}_{i} \cdot \vec{n}\right) \vec{n}$, where $\vec{n}$ is defined in Eq.(3). This is a phenomenological approach able to effectively represent all the microscopic processes responsible for the overall dipole damped motion. The two forces can be summed to give $\vec{F}_{i}=\vec{F}_{i, L}+\vec{F}_{i, D}$. In conclusion, we have to calculate the following contributions to the generalized forces

$$
\begin{aligned}
& Q_{\vartheta, L}=q_{0} \sum_{i=1}^{N} \vec{v}_{i} \wedge \vec{B} \cdot \frac{\partial \vec{r}_{i}}{\partial \vartheta}, \\
& Q_{\vartheta, D}=-k_{0} \sum_{i=1}^{N}\left(\vec{v}_{i} \cdot \vec{n}\right)\left(\vec{n} \cdot \frac{\partial \vec{r}_{i}}{\partial \vartheta}\right), \\
& Q_{\varphi, L}=q_{0} \sum_{i=1}^{N} \vec{v}_{i} \wedge \vec{B} \cdot \frac{\partial \vec{r}_{i}}{\partial \varphi}, \\
& Q_{\varphi, D}=-k_{0} \sum_{i=1}^{N}\left(\vec{v}_{i} \cdot \vec{n}\right)\left(\vec{n} \cdot \frac{\partial \vec{r}_{i}}{\partial \varphi}\right),
\end{aligned}
$$

which can be summed to give

$$
\begin{gathered}
Q_{\vartheta}=Q_{\vartheta, L}+Q_{\vartheta, D}, \\
Q_{\varphi}=Q_{\varphi, L}+Q_{\varphi, D} .
\end{gathered}
$$

While the exact expressions of the quantities in Eqs.(20)-(23) are given in Appendix A, we perform here the continuous limit of these generalized forces. By recalling that $q_{0}=q / N$ and that $d p \simeq 2 \pi / N$ in Eqs.(A1)-(A2), a straightforward integration, as before, yields the following results for the components related to the Lorentz force

$$
\begin{aligned}
Q_{\vartheta, L} & =\frac{q \omega R^{2}}{2} \vec{\lambda} \cdot \vec{B}+\frac{q \dot{\varphi} R^{2}}{2} \cos \vartheta \vec{\lambda} \cdot \vec{B} \\
Q_{\varphi, L} & =\frac{q \omega R^{2}}{2} \sin \vartheta \vec{\mu} \cdot \vec{B}-\frac{q \dot{\vartheta} R^{2}}{2} \cos \vartheta \vec{\lambda} \cdot \vec{B}
\end{aligned}
$$

where we used the definitions of $\vec{\lambda}$ and $\vec{\mu}$ given in Eqs.(4) and (5), respectively. Concerning the damping terms given in Eqs.(A3)-(A4), we define $k=N K_{0}$ and we get the continuous limit as

$$
\begin{aligned}
& Q_{\vartheta, D}=-\frac{1}{2} k R^{2} \dot{\vartheta}, \\
& Q_{\varphi, D}=-\frac{1}{2} k R^{2} \dot{\varphi} \sin ^{2} \vartheta .
\end{aligned}
$$

We have now all the explicit terms to write down the Lagrange equations for the dipole time evolution. The left hand sides are summarized in Eqs.(18) and (19) while the right hand sides are given in Eqs.(26)-(29), which can be summed as in Eqs.(24) and (25). Hence, Eqs.(7) and (8) can be finally written as

$$
\begin{aligned}
& m \omega R^{2}\left[\dot{\varphi} \sin \vartheta+\frac{1}{2 \omega}\left(\ddot{\vartheta}+\dot{\varphi}^{2} \sin \vartheta \cos \vartheta\right)\right] \\
& =\frac{q \omega R^{2}}{2}\left[\vec{\lambda} \cdot \vec{B}+\frac{\dot{\varphi}}{\omega} \cos \vartheta \vec{\lambda} \cdot \vec{B}\right]-\frac{1}{2} k R^{2} \dot{\vartheta} \\
& m \omega R^{2}\left[-\dot{\vartheta} \sin \vartheta+\frac{1}{2 \omega}\left(2 \ddot{\varphi}-\ddot{\varphi} \sin ^{2} \vartheta-2 \dot{\vartheta} \dot{\varphi} \sin \vartheta \cos \vartheta\right)\right] \\
& =\frac{q \omega R^{2}}{2}\left[\sin \vartheta \vec{\mu} \cdot \vec{B}-\frac{\dot{\vartheta}}{\omega} \cos \vartheta \vec{\lambda} \cdot \vec{B}\right]-\frac{1}{2} k R^{2} \dot{\varphi} \sin ^{2} \vartheta
\end{aligned}
$$

By straightforward simplifications, we get

$$
\begin{aligned}
& \dot{\varphi} \sin \vartheta+\frac{1}{2 \omega}\left(\ddot{\vartheta}+\dot{\varphi}^{2} \sin \vartheta \cos \vartheta\right) \\
& =\gamma\left[\vec{\lambda} \cdot \vec{B}+\frac{\dot{\varphi}}{\omega} \cos \vartheta \vec{\lambda} \cdot \vec{B}\right]-\alpha \dot{\vartheta}, \\
& -\dot{\vartheta} \sin \vartheta+\frac{1}{2 \omega}\left(2 \ddot{\varphi}-\ddot{\varphi} \sin ^{2} \vartheta-2 \dot{\vartheta} \dot{\varphi} \sin \vartheta \cos \vartheta\right) \\
& =\gamma\left[\sin \vartheta \vec{\mu} \cdot \vec{B}-\frac{\dot{\vartheta}}{\omega} \cos \vartheta \vec{\lambda} \cdot \vec{B}\right]-\alpha \dot{\varphi} \sin ^{2} \vartheta,
\end{aligned}
$$

where we introduced the gyromagnetic ratio $\gamma=\frac{q}{2 m}$ and the Gilbert damping coefficient $\alpha=\frac{k}{2 m \omega}$. This is the main achievement of the present Section and represents the set of dynamical equations for the reorientation of the magnetic dipole subjected to external magnetic field and damping. These equations have been obtained without any form of approximation, starting from the basic assumptions reported above. We further observe that three parameters $\gamma, \alpha$ and $\omega$ completely control these dynamical process.

It is important to observe that the obtained equations contain some terms that are proportional to $1 / \omega$. These terms can be explained as follows. The terms proportional to $1 / \omega$ in the left hand side of Eqs.(32) and (33) are responsible for the inertial behavior of the magnetic dipole and are indeed related to the second derivatives of precession and nutation angles $\varphi$ and $\vartheta$. On the other hand, the terms proportional to $1 / \omega$ in the right hand side of Eqs.(32) and (33) represent the Lorentz force generated by the reorientation of the dipole plane $\Pi$. Indeed, such a reorientation produces a charge velocity not related to $\omega$ but rather to $\dot{\varphi}$ and $\dot{\vartheta}$. All these kind of terms can be typically neglected for real microscopic dipole or, equivalently, for the so-called ideal dipole. An ideal magnetic dipole is indeed characterized by $\omega \rightarrow \infty$ and $R \rightarrow 0$, but with a finite value of $M=\frac{1}{2} q \omega R^{2}$. It means that we have a magnetic dipole with an infinitely small size and an infinitely large electric current, so that to have a finite dipole moment. In other words, to deal with an ideal dipole, we have to suppose that $\omega \gg \dot{\vartheta}$ and $\omega \gg \dot{\varphi}$. Equivalently, the intrinsic rotation of the charged particles is much faster than the reorientation process of the dipole plane. Concerning the damping process, the ideal dipole is characterized by the limiting values $k \rightarrow \infty$ and $\omega \rightarrow \infty$, performed by taking a finite value for the damping coefficient $\alpha=\frac{k}{2 m \omega}$.

The meaning of the ideal dipole approximation characterized by $\omega \rightarrow \infty$ can be appreciated by considering the paradigmatic magnetic dipole constituted by a hydrogen Bohr atom 
with one electron and one proton. In this case, we have $q=e=1.6 \times 10^{-19} \mathrm{C}, m=m_{e}=9.1 \times 10^{-31} \mathrm{Kg}$, and the dipole radius $R$ coincides with the Bohr radius $a_{0}$ given by

$$
a_{0}=\frac{4 \pi \varepsilon_{0} \hbar^{2}}{m_{e} e^{2}} \simeq 0.5 \times 10^{-10} \mathrm{~m} .
$$

Moreover, the Bohr theory allows the determination of the electron orbital velocity as

$$
v_{e}=\frac{e^{2}}{4 \pi \varepsilon_{0} \hbar} \simeq 2.1 \times 10^{6} \mathrm{~ms}^{-1},
$$

also corresponding to the fine-structure constant $(\sim 1 / 137)$ times the speed of light in vacuum $c=299792458 \mathrm{~m} / \mathrm{s}$. The Bohr radius and the electron velocity can be used to directly calculate the angular frequency as follows

$$
\omega=\frac{v_{e}}{a_{0}}=\frac{m_{e} e^{4}}{\left(4 \pi \varepsilon_{0}\right)^{2} \hbar^{3}} \simeq 4.2 \times 10^{16} \mathrm{~s}^{-1} .
$$

Therefore, we observe that for this magnetic dipole, $\omega$ assumes a very large value, confirming the validity of the ideal dipole hypothesis. To conclude, we can also determine the dipole moment of the electron rotation as

$$
M=\frac{e v_{e} a_{0}}{2}=\frac{e \hbar}{2 m_{e}} \simeq 9.2 \times 10^{-24} \mathrm{Am}^{2},
$$

corresponding to the so-called Bohr magneton. We can state that for such a system, the ideal dipole approximation is valid if the frequency $f$ of the applied magnetic induction $\vec{B}$ is much lower than $\omega /(2 \pi) \sim 10^{16} \mathrm{~s}^{-1}$. Consequently, the inertial effect in the magnetization reorientation can be appreciated only with very large frequencies of the applied magnetic field. We remark that neglecting the terms of the order $1 / \omega$ in Eqs.(32) and (33) transforms the second order Lagrange equations in a set of first order differential equation. This is coherent with the classical forms of the LLG equation, as generally used in micromagnetism $[26,27]$. As discussed in the Introduction, the problem of the inertial effect in the dynamics of magnetization has been investigated in recent literature [45-55]. In these works, an evolution equation for the magnetization has been proposed. However, it is not completely consistent with our Eqs.(32) and (33). Indeed, in the previously proposed equation, the terms corresponding to the Lorentz force generated by the reorientation dynamics [our $1 / \omega$ terms in the r.h.s. of Eqs.(32) and (33)] have been completely neglected and the purely inertial terms are similar but not coinciding with ours. The origin of the differences between our approach and previous works is due to the fact that in Refs.[45-55] the intrinsic rotational motion of the charge defining the magnetic dipole is not considered as a basic assumption and therefore the inertial and Lorentz forces are introduced in a different way. An alternative approach useful to better draw a comparison with the equation proposed by Wegrowe and co-workers is discussed in the next Section.

Anyway, if we neglect the terms of the order of $1 / \omega$ in Eqs.(32) and (33), we get the simplified relations

$$
\begin{aligned}
\dot{\varphi} \sin \vartheta & =\gamma \vec{\lambda} \cdot \vec{B}-\alpha \dot{\vartheta} \\
\dot{\vartheta} & =-\gamma \vec{\mu} \cdot \vec{B}+\alpha \sin \vartheta \dot{\varphi}
\end{aligned}
$$

This is a first-order system of differential equations, which is not written in normal form. To obtain its normal form, we can substitute $\dot{\vartheta}$ from Eq.(39) into Eq.(38) and, reciprocally, $\dot{\varphi} \sin \vartheta$ from Eq.(38) into Eq.(39). This procedure eventually yields

$$
\begin{aligned}
\dot{\varphi} \sin \vartheta & =\frac{\gamma}{1+\alpha^{2}}(\vec{\lambda} \cdot \vec{B}+\alpha \vec{\mu} \cdot \vec{B}), \\
\dot{\vartheta} & =\frac{\gamma}{1+\alpha^{2}}(\alpha \vec{\lambda} \cdot \vec{B}-\vec{\mu} \cdot \vec{B}) .
\end{aligned}
$$

These polar forms of the equations for the magnetization dynamics have been largely used in different applications $[62,63]$. To conclude, it is not difficult to prove that Eqs.(38) and (39) are equivalent to the first form (implicit) of the LLG equation

$$
\frac{d \vec{M}}{d t}=\gamma \vec{M} \wedge \vec{B}-\frac{\alpha}{M} \vec{M} \wedge \frac{d \vec{M}}{d t},
$$

while Eqs.(40) and (41) are equivalent to the second form (explicit) of the LLG equation

$$
\frac{d \vec{M}}{d t}=\frac{\gamma}{1+\alpha^{2}}\left[\vec{M} \wedge \vec{B}-\frac{\alpha}{M} \vec{M} \wedge(\vec{M} \wedge \vec{B})\right] .
$$

To directly prove these equivalences, it is sufficient to consider that $\vec{M}=M \vec{n}$ and use the definition of $\vec{n}$ given in Eq.(3). From this result we deduce that the Gilbert damping process appears to be more adapted to describe the magnetization dynamics than the Landau-Lifshitz counterpart since it has been obtained from a purely mechanical model. Notice that with the above formulation, it is difficult to close the equations of motion for $\vec{n}$ accounting for inertial effects when $\omega \rightarrow \infty$, as all second order derivatives are, at first glance, wiped out by such a limiting process. Thus, the purpose of the next section is to demonstrate that, in the same limit $\omega \rightarrow \infty$, a closedform equation for $\vec{n}$ can be found that includes second-order derivatives, and therefore of magnetic inertial effects similar with those which have been experimentally evidenced recently [55].

\section{MAGNETIC INERTIA CORRECTED GILBERT EQUATION FROM THE CIRCULAR LOOP MODEL}

The main equations derived in the previous Section, namely Eqs.(32) and (33) are able to describe all effects produced by an external magnetic field on a magnetic dipole. However, as alluded to in the previous section their form is not symmetric and it is difficult, if not impossible, to obtain a dynamic equation written only in terms of the vector $\vec{n}$ or $\vec{M}$, as expected to get a generalization of the classical LLG equation. So, we describe here an alternative approach eventually yielding a more symmetric formalism and giving a rigorous justification of the Wegrowe equation.

As before, we consider the moving frame defined by $(\vec{n}, \vec{\lambda}, \vec{\mu})$, constituting a convenient basis which is rigidly bound with the rotating loop. Now, the rotational frequency 
of the charges is arbitrarily varying and we can write their positions as

$$
\vec{r}_{i}(t)=\vec{\lambda} R \cos \left(\psi(t)+p_{i}\right)+\vec{\mu} R \sin \left(\psi(t)+p_{i}\right),
$$

where $\psi$ represents the arbitrary angular velocity of the charges. Notice that here, the constraints are holonomic and time-independent. However, this does not fundamentally change the basic analysis conducted in the previous section. The kinetic energy of the system in the form

$$
T=\frac{m R^{2}}{4}\left(\dot{\vartheta}^{2}+\dot{\varphi}^{2} \sin ^{2} \vartheta\right)+\frac{m R^{2}}{2}(\dot{\psi}+\dot{\varphi} \cos \vartheta)^{2},
$$

which is consistent with Eq.(17) by replacing $\psi$ by $\omega$ in Eq.(45). Here again, we introduced $m=N m_{0}$ and we performed the continuum limit, in exactly the same fashion as discussed in the previous section. Written in this form, the kinetic energy is identical to that of a symmetric top with one point fixed, with principal moments of inertia $I_{1}=I_{2}=m R^{2} / 2$ and $I_{3}=m R^{2}$ [61]. This point definitively shows that the classical mechanics is able to mimic the magnetization dynamics with three positive moments of inertia. Now, the generalized coordinates are three in number, namely $\left(q_{1}, q_{2}, q_{3}\right)=$ $(\varphi, \vartheta, \psi)$ as it must for a symmetric top with one point fixed, and the generalized velocities are $\left(\dot{q}_{1}, \dot{q}_{2}, \dot{q}_{3}\right)=(\dot{\varphi}, \dot{\vartheta}, \dot{\psi})$. Before writing the equations of motion, we also write the total force to which the charges are subjected. This is given by the Lorentz force combined with the damping force

$$
\vec{F}_{i}=\vec{F}_{i, L}+\vec{F}_{i, D}=q_{0} \vec{v}_{i} \wedge \vec{B}-k_{0}\left(\vec{v}_{i} \cdot \vec{n}\right) \vec{n} .
$$

Then, we write the Lagrange equations as [61]

$$
\frac{d}{d t} \frac{\partial T}{\partial \dot{q}_{k}}-\frac{\partial T}{\partial q_{k}}=\sum_{i=1}^{N} \vec{F}_{i} \cdot \frac{\partial \vec{r}_{i}}{\partial q_{k}}, \quad k=1,2,3 .
$$

By introducing the gyromagnetic ratio $\gamma=\frac{q}{2 m}=\frac{q_{0}}{2 m_{0}}$, the apparent damping constant $k=N k_{0}$ and using the discrete to continuous limit to evaluate the sums in Eq.(47), we explicitly obtain the Lagrange equations

$$
\begin{aligned}
& \frac{d}{d t}\left(2 \dot{\varphi}-\dot{\varphi} \sin ^{2} \vartheta+2 \dot{\psi} \cos \vartheta\right) \\
& =2 \gamma \dot{\psi} \sin \vartheta \vec{\mu} \cdot \vec{B}-2 \gamma \dot{\vartheta} \cos \vartheta \vec{\lambda} \cdot \vec{B}-\frac{k}{m} \dot{\varphi} \sin ^{2} \vartheta \\
& \ddot{\vartheta}+\dot{\varphi} \sin \vartheta(\dot{\varphi} \cos \vartheta+2 \dot{\psi}) \\
& =2 \gamma \dot{\psi} \vec{\lambda} \cdot \vec{B}+2 \gamma \dot{\varphi} \cos \vartheta \vec{\lambda} \cdot \vec{B}-\frac{k}{m} \dot{\vartheta}
\end{aligned}
$$

and

$$
\frac{d}{d t}(\dot{\psi}+\dot{\varphi} \cos \vartheta)=-\gamma \dot{\vartheta} \vec{\lambda} \cdot \vec{B}-\gamma \dot{\varphi} \sin \vartheta \vec{\mu} \cdot \vec{B}
$$

We set now

$$
\Omega=\dot{\psi}+\dot{\varphi} \cos \vartheta
$$

From Eq.(50), we may write

$$
\gamma \dot{\vartheta} \vec{\lambda} \cdot \vec{B}=-\gamma \dot{\varphi} \sin \vartheta \vec{\mu} \cdot \vec{B}-\dot{\Omega}
$$

and this result can be substituted in the first Lagrange equation given in Eq.(48). After straightforward algebra we get

$$
\begin{gathered}
\ddot{\varphi} \sin \vartheta+2 \dot{\vartheta} \dot{\varphi} \cos \vartheta-2 \Omega \dot{\vartheta}=-\frac{k}{m} \dot{\varphi} \sin \vartheta+2 \gamma \Omega \vec{\mu} \cdot \vec{B}, \\
\ddot{\vartheta}-\dot{\varphi}^{2} \sin \vartheta \cos \vartheta+2 \Omega \dot{\varphi} \sin \vartheta=-\frac{k}{m} \dot{\vartheta}+2 \gamma \Omega \vec{\lambda} \cdot \vec{B},
\end{gathered}
$$

while we also have Eq.(50), viz.

$$
\dot{\Omega}=-\gamma \dot{\vartheta} \vec{\lambda} \cdot \vec{B}-\gamma \dot{\varphi} \sin \vartheta \vec{\mu} \cdot \vec{B}
$$

In order to handle the inertial terms, we consider now the vector $\vec{J}$ defined by

$$
\begin{aligned}
\vec{J} & =\vec{n} \wedge \frac{d^{2} \vec{n}}{d t^{2}} \\
& =\left(\ddot{\vartheta}-\dot{\varphi}^{2} \sin \vartheta \cos \vartheta\right) \vec{\mu}-(\ddot{\varphi} \sin \vartheta+2 \dot{\vartheta} \dot{\varphi} \cos \vartheta) \vec{\lambda} \\
& =J_{\mu} \vec{\mu}+J_{\lambda} \vec{\lambda}
\end{aligned}
$$

Then, we may write Eqs.(53) and (54) as

$$
\begin{gathered}
-J_{\lambda}-2 \Omega \dot{\vartheta}=-\frac{k}{m} \dot{\varphi} \sin \vartheta+2 \gamma \Omega \vec{\mu} \cdot \vec{B}, \\
J_{\mu}+2 \Omega \dot{\varphi} \sin \vartheta=-\frac{k}{m} \dot{\vartheta}+2 \gamma \Omega \vec{\lambda} \cdot \vec{B} .
\end{gathered}
$$

This form exhibits a complete symmetry and can be further developed as follows

$$
\begin{aligned}
& -J_{\lambda}-2 \dot{\psi}\left(1+\frac{\dot{\varphi} \cos \vartheta}{\dot{\psi}}\right) \dot{\vartheta} \\
& =-\frac{k}{m} \dot{\varphi} \sin \vartheta+2 \gamma \dot{\psi}\left(1+\frac{\dot{\varphi} \cos \vartheta}{\dot{\psi}}\right) \vec{\mu} \cdot \vec{B} \\
& J_{\mu}+2 \dot{\psi}\left(1+\frac{\dot{\varphi} \cos \vartheta}{\dot{\psi}}\right) \dot{\varphi} \sin \vartheta \\
& =-\frac{k}{m} \dot{\vartheta}+2 \gamma \dot{\psi}\left(1+\frac{\dot{\varphi} \cos \vartheta}{\dot{\psi}}\right) \vec{\lambda} \cdot \vec{B} .
\end{aligned}
$$

These equations must be combined with Eq.(50). Indeed, we remark that Eqs.(59) and (60) completely describe the motion of $\vec{n}$, the unit normal to the loop, if the dynamics of $\psi$ is known. However, for the description of the magnetic moment dynamics, we can consider the value of $\psi$ large (with respect to $\dot{\vartheta}$ and $\dot{\varphi}$ ) and constant since the modulus of the magnetic moment and the damping coefficient should be considered as constant parameters. This is accomplished if

$$
\dot{\psi}=\omega
$$

where $\omega$ is a constant (the same considered in the previous Section). This choice is actually legitimate since the damping force does not play any role in Eq.(50). With this hypothesis, 
the motion of $\vec{n}$ is governed by the couple of equations

$$
\begin{aligned}
& -J_{\lambda}-2 \omega\left(1+\frac{\dot{\varphi} \cos \vartheta}{\omega}\right) \dot{\vartheta} \\
& =-\frac{k}{m} \dot{\varphi} \sin \vartheta+2 \gamma \omega\left(1+\frac{\dot{\varphi} \cos \vartheta}{\omega}\right) \vec{\mu} \cdot \vec{B}, \\
& J_{\mu}+2 \omega\left(1+\frac{\dot{\varphi} \cos \vartheta}{\omega}\right) \dot{\varphi} \sin \vartheta \\
& =-\frac{k}{m} \dot{\vartheta}+2 \gamma \omega\left(1+\frac{\dot{\varphi} \cos \vartheta}{\omega}\right) \vec{\lambda} \cdot \vec{B},
\end{aligned}
$$

where $\omega$ is a constant representing the angular frequency of the charges rotation (nutation frequency). The set of Eqs.(62) and (63) represents our second proposed model for the magnetization dynamics. Its form is more elegant and symmetric than the one given in Eqs.(32) and (33). Moreover, it allows to draw a comparison with the dynamic equation recently proposed by Wegrowe and co-workers.

Actually, a further simplification can be introduced by assuming that

$$
\dot{\varphi} \cos \vartheta \ll \omega \quad \text { or } \quad \dot{\varphi} \ll \omega .
$$

The last two equations become

$$
\begin{array}{r}
-J_{\lambda}-2 \omega \dot{\vartheta}=-\frac{k}{m} \dot{\varphi} \sin \vartheta+2 \gamma \omega \vec{\mu} \cdot \vec{B}, \\
J_{\mu}+2 \omega \dot{\varphi} \sin \vartheta=-\frac{k}{m} \dot{\vartheta}+2 \gamma \omega \vec{\lambda} \cdot \vec{B} .
\end{array}
$$

Finally, redefining the dimensionless damping constant

$$
\alpha=\frac{k}{2 m \omega},
$$

and introducing the time constant $\tau$ by

$$
\tau=\frac{1}{2 \omega}
$$

we easily obtain

$$
\begin{array}{r}
-\tau J_{\lambda}-\dot{\vartheta}=-\alpha \dot{\varphi} \sin \vartheta+\gamma \vec{\mu} \cdot \vec{B}, \\
\tau J_{\mu}+\dot{\varphi} \sin \vartheta=-\alpha \dot{\vartheta}+\gamma \vec{\lambda} \cdot \vec{B},
\end{array}
$$

or, equivalently, the equation for $\vec{n}$ in the form

$$
\frac{d \vec{n}}{d t}=\gamma \vec{n} \wedge \vec{B}-\alpha \vec{n} \wedge \frac{d \vec{n}}{d t}-\tau \vec{n} \wedge \frac{d^{2} \vec{n}}{d t^{2}},
$$

which is equivalent to Eqs.(65) and (66) and represents the equation of motion for the magnetic dipole proposed in recent literature by Wegrowe and co-workers [45-50]. Of course, in the noninertial limit defined by $\omega \rightarrow \infty$ or $\tau \rightarrow 0$, we obtain again the simplified form

$$
\frac{d \vec{n}}{d t}=\gamma \vec{n} \wedge \vec{B}-\alpha \vec{n} \wedge \frac{d \vec{n}}{d t},
$$

which is the Gilbert's equation for the dynamics of the magnetization direction. As a conclusion, we can state that the equation of Wegrowe and co-workers can be obtained as an approximation (with $\dot{\varphi} \ll \omega$ ) of the exact equations of motion governing the dynamics of a circular current loop, in turn given by that of a symmetric top with one point fixed with well-identified moments of inertia.

\section{FREQUENCY RESPONSE}

We investigate now the characteristic frequency response corresponding to the proposed models, since it is the feature typically investigated with standard experimental approaches. It means that we apply a uniform and constant bias field $\vec{B}_{0}$ to the magnetic dipole, with an additive time-varying small perturbation $\delta \vec{B}$, and we observe the resulting dipole motion. To simplify the notation, we define the vector $\vec{x}=(\varphi, \vartheta)$, describing the magnetization orientation. In response to the applied field, we can observe a preferential fixed direction identified by $\vec{x}_{0}=\left(\varphi_{0}, \vartheta_{0}\right)$, perturbed by a small time-varying quantity $\delta \vec{x}$. In previous Sections, we discussed three different versions of the equations describing the noninertial dynamics of magnetization: (i) Eqs.(32) and (33), obtained by considering a uniformly rotating distribution of charge; (ii) Eqs.(62) and (63), obtained through a symmetric top with one point fixed; (iii) Eqs.(69) and (70), which represent a simplification of the second form for high values of $\omega$ (coinciding with the equation proposed by Wegrowe and co-workers). These three sets of equations can be cast into the following general form

$$
\begin{aligned}
& f_{1}(\vec{x}, \dot{\vec{x}}, \ddot{\vec{x}}, \vec{B})=0, \\
& f_{2}(\vec{x}, \dot{\vec{x}}, \ddot{\vec{x}}, \vec{B})=0,
\end{aligned}
$$

where $f_{1}$ and $f_{2}$ are suitable functions representing any of the three models above. We describe here an ad hoc procedure of linearization for this arbitrary system of differential equations. To begin, we can substitute the assumed hypotheses $\vec{x}=\vec{x}_{0}+$ $\delta \vec{x}$ and $\vec{B}=\vec{B}_{0}+\delta \vec{B}$ in Eqs.(73) and (74), eventually obtaining

$$
\begin{aligned}
& f_{1}\left(\vec{x}_{0}+\delta \vec{x}, \delta \dot{\vec{x}}, \delta \ddot{\vec{x}}, \vec{B}_{0}+\delta \vec{B}\right)=0, \\
& f_{2}\left(\vec{x}_{0}+\delta \vec{x}, \delta \dot{\vec{x}}, \delta \ddot{\vec{x}}, \vec{B}_{0}+\delta \vec{B}\right)=0 .
\end{aligned}
$$

Since the applied perturbation $\delta \vec{B}$ and the resulting perturbation $\delta \vec{x}$ are supposed to be small with respect to $\vec{B}_{0}$ and $\vec{x}_{0}$, respectively, we can develop previous equations to the first order as follows

$$
\begin{aligned}
f_{1}\left(\vec{x}_{0}, 0,0, \vec{B}_{0}\right) & +\frac{\partial f_{1}}{\partial \vec{x}} \cdot \delta \vec{x}+\frac{\partial f_{1}}{\partial \dot{\vec{x}}} \cdot \delta \dot{\vec{x}} \\
& +\frac{\partial f_{1}}{\partial \ddot{\vec{x}}} \cdot \delta \ddot{\vec{x}}+\frac{\partial f_{1}}{\partial \overrightarrow{\vec{B}}} \cdot \delta \vec{B}=0, \\
f_{2}\left(\vec{x}_{0}, 0,0, \vec{B}_{0}\right) & +\frac{\partial f_{2}}{\partial \vec{x}} \cdot \delta \vec{x}+\frac{\partial f_{2}}{\partial \dot{\vec{x}}} \cdot \delta \dot{\vec{x}} \\
& +\frac{\partial f_{2}}{\partial \ddot{\vec{x}}} \cdot \delta \ddot{\vec{x}}+\frac{\partial f_{2}}{\partial \vec{B}} \cdot \delta \vec{B}=0,
\end{aligned}
$$

where the partial derivatives are calculated for $\vec{x}=\vec{x}_{0}, \dot{\vec{x}}=0$, $\ddot{\vec{x}}=0$ and $\vec{B}=\vec{B}_{0}$. Now, we clearly have that $f_{1}\left(\vec{x}_{0}, 0,0, \vec{B}_{0}\right)=$ 0 and $f_{2}\left(\vec{x}_{0}, 0,0, \vec{B}_{0}\right)=0$, since $\vec{x}_{0}$ is the magnetization direction induced by $\vec{B}_{0}$ when the perturbations are not applied. To make this procedure more effective, we suppose that the perturbation of the applied magnetic induction is given by the sinusoidal oscillation

$$
\delta \vec{B}=\operatorname{Re}\left\{\vec{b} e^{i \Omega_{B} t}\right\},
$$

where $\Omega_{B}$ is the angular frequency and $\vec{b}$ is the corresponding complex amplitude (phasor). Here $\operatorname{Re}\{z\}$ stands for the 
real part of the complex number $z$. Of course, also the angles perturbation follows a similar time evolution

$$
\delta \vec{x}=\operatorname{Re}\left\{\vec{a} e^{i \Omega_{B} t}\right\},
$$

with the same angular frequency and where $\vec{a}$ is its complex amplitude (phasor). It means that we are in a sinusoidal steady state regime. While the applied phasor $\vec{b}=\left(b_{x}, b_{y}, b_{z}\right) \in \mathbb{C}^{3}$ is known, the resulting phasor $\vec{a}=\left(a_{\varphi}, a_{\vartheta}\right) \in \mathbb{C}^{2}$ is unknown and it can be determined as follows. By using Eqs.(79) and (80) in Eqs.(77) and (78), we easily obtain

$$
\begin{aligned}
& \left(\frac{\partial f_{1}}{\partial \vec{x}}+i \Omega_{B} \frac{\partial f_{1}}{\partial \dot{\vec{x}}}-\Omega_{B}^{2} \frac{\partial f_{1}}{\partial \ddot{\vec{x}}}\right) \cdot \vec{a}+\frac{\partial f_{1}}{\partial \vec{B}} \cdot \vec{b}=0, \\
& \left(\frac{\partial f_{2}}{\partial \vec{x}}+i \Omega_{B} \frac{\partial f_{2}}{\partial \dot{\vec{x}}}-\Omega_{B}^{2} \frac{\partial f_{2}}{\partial \overrightarrow{\vec{x}}}\right) \cdot \vec{a}+\frac{\partial f_{2}}{\partial \vec{B}} \cdot \vec{b}=0,
\end{aligned}
$$

which is a system of two linear equations in the two unknown components of the vector $\vec{a}$. This vector $\vec{a}$ can be simply obtained by calculating all the partial derivatives needed in Eqs.(81) and (82), starting from the mathematical expressions of $f_{1}$ and $f_{2}$, and by solving the linear system. To simplify the calculation, we fix $\vec{B}_{0}=(B, 0,0)$, i.e. we suppose the the bias filed is applied to the $x$-direction of the reference frame. Of course, this assumption does not limit the generality of the following achievements. The interesting point is that we get exactly the same result for the three models proposed and discussed previously. It means that Eqs.(32) and (33), Eqs.(62) and (63), and Eqs.(69) and (70) yield the same vector $\vec{a}$ given by

$$
\begin{aligned}
& a_{\varphi}=\frac{2 \omega \gamma\left(2 i \Omega_{B} \omega b_{z}-2 b_{y} \gamma B \omega-2 i b_{y} \Omega_{B} \alpha \omega+b_{y} \Omega_{B}^{2}\right)}{\mathscr{D}}, \\
& a_{\vartheta}=\frac{2 \omega \gamma\left(2 i \Omega_{B} \omega b_{y}+2 b_{z} \gamma B \omega+2 i b_{z} \Omega_{B} \alpha \omega-b_{z} \Omega_{B}^{2}\right)}{\mathscr{D}},
\end{aligned}
$$

where

$$
\begin{aligned}
\mathscr{D}= & 4 \Omega_{B}^{2} \omega^{2}-4 \gamma^{2} B^{2} \omega^{2}-8 i \gamma B \omega^{2} \Omega_{B} \alpha \\
& +4 \gamma B \omega \Omega_{B}^{2}+4 \Omega_{B}^{2} \alpha^{2} \omega^{2}+4 i \Omega_{B}^{3} \alpha \omega-\Omega_{B}^{4},
\end{aligned}
$$

which is a fourth degree polynomial in the applied angular frequency $\Omega_{B}$.

The quantities $a_{\vartheta}$ and $a_{\vartheta}$ are used as follows to obtain the fluctuations of the direction $\vec{n}$. To begin, we can write $\vec{n}=$ $\vec{n}_{0}+\delta \vec{n}$, where $\vec{n}_{0}$ is identified by $\vec{x}_{0}=\left(\varphi_{0}, \vartheta_{0}\right)$. Concerning the perturbation we can assume that

$$
\delta \vec{n}=\operatorname{Re}\left\{\vec{v} e^{i \Omega_{B} t}\right\}
$$

where $\vec{v}$ is the complex amplitude associated to $\delta \vec{n}$. Then, a simple use of Eq.(3) leads to the first order relations

$$
\begin{aligned}
& v_{x}=-\sin \varphi_{0} \sin \vartheta_{0} a_{\varphi}+\cos \varphi_{0} \cos \vartheta_{0} a_{\vartheta} \\
& v_{y}=\cos \varphi_{0} \sin \vartheta_{0} a_{\varphi}+\sin \varphi_{0} \cos \vartheta_{0} a_{\vartheta}, \\
& v_{z}=-\sin \vartheta_{0} a_{\vartheta} .
\end{aligned}
$$

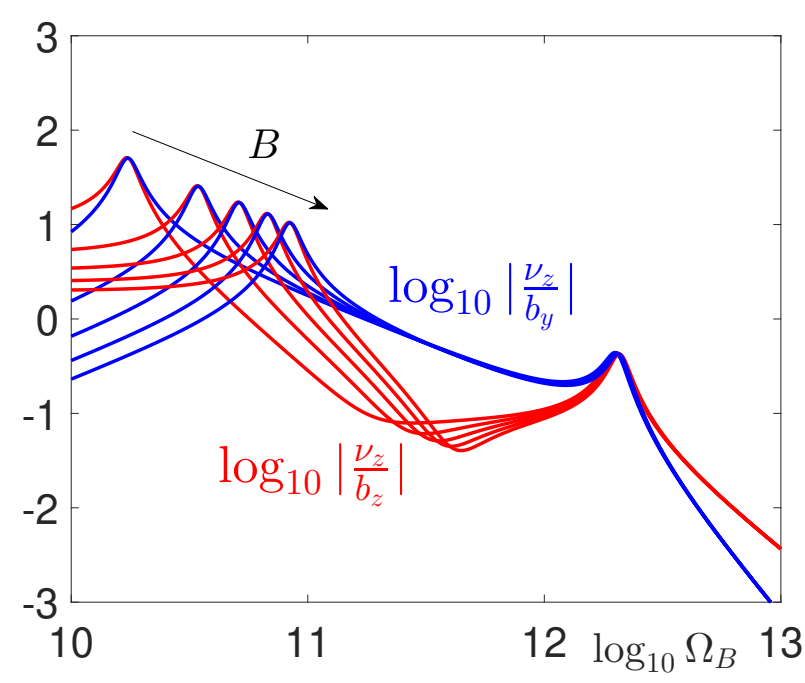

FIG. 3. Frequency response of the system with a varying value of the applied magnetic induction $B$. We adopted the parameters $\gamma=$ $1.76 \times 10^{11} \mathrm{~s}^{-1} \mathrm{~T}^{-1}, B=0.1,0.2,0.3,0.4,0.5 \mathrm{~T}, \alpha=0.1$ and $\omega=1 \times$ $10^{12} \mathrm{~s}^{-1}$. The arrow indicates the increasing values of $B$.

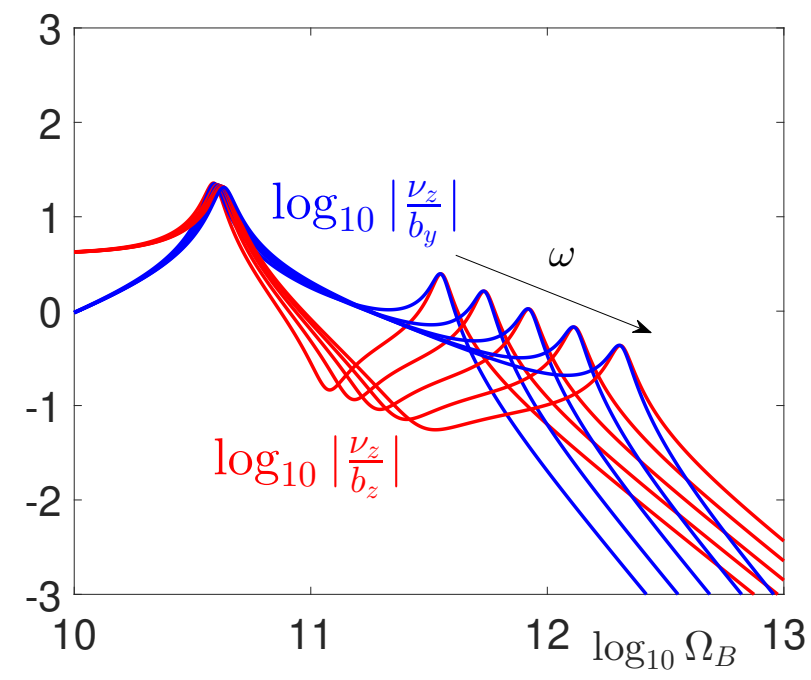

FIG. 4. Frequency response of the system with a varying value of the intrinsic frequency $\omega$. We adopted the parameters $\gamma=1.76 \times$ $10^{11} \mathrm{~s}^{-1} \mathrm{~T}^{-1}, B=0.25 \mathrm{~T}, \alpha=0.1$ and $\omega=1.6 \times 10^{11}, 2.5 \times 10^{11}, 4 \times$ $10^{11}, 6.3 \times 10^{11}, 1 \times 10^{12} \mathrm{~s}^{-1}$. The arrow indicates the increasing values of $\omega$.

Therefore, we obtain from Eqs.(83) and (84) the following simplified expressions based on the assumption $\vec{B}_{0}=(B, 0,0)$

$$
\begin{aligned}
& v_{x}=0, \\
& v_{y}=a_{\varphi}, \\
& v_{z}=-a_{\vartheta} .
\end{aligned}
$$

We study the behavior of $v_{z}$ when only $b_{y} \neq 0$ and when only 


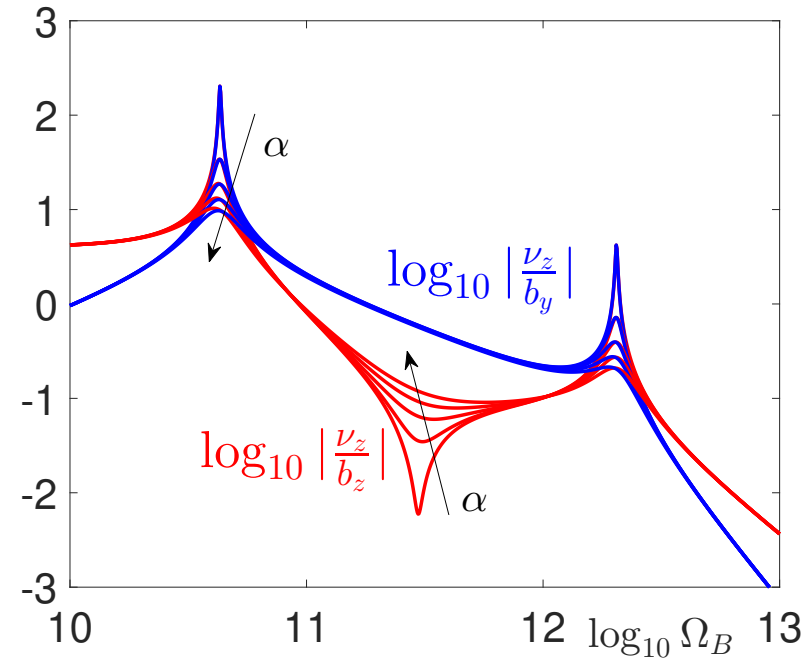

FIG. 5. Frequency response of the system with a varying value of the damping factor $\alpha$. We adopted the parameters $\gamma=1.76 \times$ $10^{11} \mathrm{~s}^{-1} \mathrm{~T}^{-1}, B=0.25 \mathrm{~T}, \alpha=0.01,0.06,0.11,0.16,0.21$ and $\omega=$ $1 \times 10^{12} \mathrm{~s}^{-1}$. The arrow indicates the increasing values of $\alpha$.

$b_{z} \neq 0$ and we eventually get

$$
\begin{aligned}
& \left.\frac{v_{z}}{b_{y}}\right|_{b_{x}=b_{z}=0}=-\frac{4 i \gamma \omega^{2} \Omega_{B}}{\mathscr{D}}, \\
& \left.\frac{v_{z}}{b_{z}}\right|_{b_{x}=b_{y}=0}=-\frac{2 \omega \gamma\left(2 \gamma B \omega+2 i \Omega_{B} \alpha \omega-\Omega_{B}^{2}\right)}{\mathscr{D}},
\end{aligned}
$$

where $\mathscr{D}$ is the polynomial defined in Eq.(85). These results represent the frequency response of the system and they are shown in Figs.3,4 and 5. In all plots we can see a first resonance that can be identified with the classical ferromagnetic resonance and a second resonance that can be ascribed to the inertial effects taken into consideration in our models. Indeed, in Fig.3, we can observe that only the first resonance frequency is shifted with an increasing polarizing field $B$, which is the classical behavior of the ferromagnetic resonance. Moreover, from Fig.4, we deduce that only the second resonance is shifted with an increasing value of $\omega$, which is the characteristic frequency describing the inertial effects. Finally, in Fig.5, we can observe the effect of the damping factor on the resonances behavior and we conclude that a smaller damping induces sharper resonance mechanism while a larger damping produces a smoother resonance response. This is true for both the ferromagnetic and the inertial resonances. The existence of the second resonance peak due to the inertial effect has been experimentally confirmed in Ref.[55], where it has been observed in ferromagnetic thin films at a frequency of approximately $0.6 \mathrm{THz}$.

The fact that the three studied models exhibit exactly the same frequency response means that the mathematical differences among them do not generate different physical behaviors. This is true, at least, for the results concerning the resonance behavior of the frequency response. A further analysis should be conducted in order to compare the complete time evolution of the magnetization for the three models with experimental data. We leave this point to further investigations.

\section{CONCLUSIONS}

In this work we readdressed the problem of mimicking the dynamics of a magnetic dipole subjected to a damping force and an external magnetic field from purely classical concepts. While the classical approaches are based on the Landau-Lifshitz equation and on its Gilbert refinement, recent experimental and theoretical investigations have shown the need to extend these theories to include inertial effects. To this aim, we propose here to consider a magnetic dipole as a circular current loop and we obtain its quantitative description through the Lagrangian mechanics.

It is important to place this dipole structure in the context of previous approaches. The idea of using the mechanical analogy between a magnetic dipole and a spinning top has been efficiently developed by Gilbert in order to derive the equation that bears his name [5,6]. The corresponding dipole can be called Gilbert magnetic dipole in order to be distinguished from the Ampère magnetic dipole. This latter is defined by a loop in which the electric current is confined (see e.g. Ref.[50]). This dichotomy has been proposed by D. J. Griffiths [64], who proved that the two dipoles are equivalent in the sub-relativistic regime of the electromagnetism. The dipole structure proposed here is a sort of intermediate version of the two systems above. Indeed, we exploited the current loop of the Ampère dipole combined with the possibility to rotate its plane through external actions, as in the Gilbert case. In this sense, the electromagnetic and the mechanical behavior are coupled to eventually obtain the dynamic equation with the inertial effects. While the Ampère magnetic dipole is classically used to determine the magnetic field produced by a dipole (by defining the dipole moment $M=I S$ ), here we use the current loop to evaluate the forces applied from an external magnetic field to the dipole itself. Since our dipole is free to rotate, these forces produce the reorientation, whose dynamics can be studied by the classical mechanical laws. We can also remark that the idea of merging the Gilbert and Ampère visions can open new perspectives concerning the full electromagnetic and mechanical analysis of the problem (based on the Maxwell and Lagrange equations). As a matter of fact, the Lagrangian function for the system can be adopted in the context of the electrodynamics in order to study the most general time-dependent situation.

Here, we followed two different lines. In the first one, we supposed a constant angular frequency for the electric charges rotating in the loop and we dealt with a rheonomic system with two degrees of freedom. In the second one, we supposed an arbitrary angular frequency for the charges by obtaining a holonomic and time-independent system with three degrees of freedom. In both cases we firstly introduced a discrete distribution of charges and we performed the limit towards a continuous structure in a second step. The effect of the magnetic field is directly introduced through the Lorentz force without using the magnetic scalar potential. Moreover, the dissipa- 
tive process is defined by a specific damping force without the need to introduce a Rayleigh dissipation function, but rather asking for this phenomenological damping force not to brake the orbital motion of the charges inside the loop (so that the current intensity inside the loop is maintained constant). This point allows a better understanding of the dissipative mechanism with a clearer definition of the force that opposes the orientation of the magnetic dipole in an externally applied magnetic field. Importantly, the proposed models naturally lead to extra terms with respect the classical LLG equation, characterizing two important phenomena: (i) the inertial effect that can be observed for high values of the frequency of the applied magnetic field; (ii) the effect of the Lorentz force generated by the reorientation of the dipole plane, which is usually neglected in previous models. While the first proposed model, with constant angular frequency of the charges, contains these two terms, its mathematical form is not symmetric and it is therefore difficult to draw a comparison with the results of the recent literature. For this reason, we introduced a refined treatment, with an arbitrary angular frequency, and we obtained a more elegant and symmetric form for the dynamical equations. Moreover, its approximation, obtained for a reasonably slow reorientation motion with respect to the rotation of charges, is found to be coinciding with the equation recently proposed by Wegrove and co-workers. Our analysis represents therefore an independent derivation of this equation. The importance of the inertial effect can be appreciated by taking into account the frequency response of the dipole system. We provided evidence that both the first and second model proposed, and also the Wegrowe equation, lead to exactly the same mathematical form of the frequency response. On the one hand, this proves that the differences between the mathematical details of the three models have no observable consequences on the physical response of the system. On the other hand, the frequency response is characterized by two resonance phenomena: while the first represents the classical ferromagnetic resonance, the second is induced by inertial effects. It is important to note that this second resonance (between $10^{11}$ and $10^{12} \mathrm{~Hz}$ ) has been observed experimentally only recently [55]. If we consider a sufficiently low applied magnetic field frequency, the proposed models can be approximated by neglecting inertial effects and provide Gilbert's equation as a result. Therefore, we can also state that a purely mechanical approach to the problem of the dynamics of a magnetic dipole gives a strong indication that the damping process is better represented by the Gilbert assumption than the Landau-Lifshitz counterpart. Finally, we clarified that the motion of a symmetric top with a fixed point, and with three positive moments of inertia, is coherent with the LLG dynamics of a magnetic dipole.

\section{Appendix A: Generalized forces}

We show here the complete expressions of the generalized forces defined in Eqs.(20)-(23), which can be summed as in
Eqs.(24) and (25). The results shown here concern the case of a discrete distribution of charged within the loop. Concerning the Lorentz force, a long but straightforward calculation leads to the expressions

$$
\begin{aligned}
Q_{\vartheta, L}= & q_{0} \omega R^{2} \sum_{i=1}^{N}\left[-B_{z} \sin \vartheta \cos ^{2}\left(\omega t+p_{i}\right)\right. \\
& +B_{y} \cos \vartheta \sin \varphi \cos ^{2}\left(\omega t+p_{i}\right) \\
& +B_{y} \cos \varphi \sin \left(\omega t+p_{i}\right) \cos \left(\omega t+p_{i}\right) \\
& +B_{x} \cos \vartheta \cos \varphi \cos ^{2}\left(\omega t+p_{i}\right) \\
& \left.-B_{x} \sin \varphi \sin \left(\omega t+p_{i}\right) \cos \left(\omega t+p_{i}\right)\right] \\
& +q_{0} \dot{\varphi} R^{2} \sum_{i=1}^{N}\left[-B_{z} \sin \vartheta \cos \vartheta \cos ^{2}\left(\omega t+p_{i}\right)\right. \\
& +B_{y} \cos { }^{2} \vartheta \sin \varphi \cos { }^{2}\left(\omega t+p_{i}\right) \\
& +B_{y} \cos \varphi \cos \vartheta \sin \left(\omega t+p_{i}\right) \cos \left(\omega t+p_{i}\right) \\
& +B_{x} \cos { }^{2} \vartheta \cos \varphi \cos ^{2}\left(\omega t+p_{i}\right) \\
& \left.-B_{x} \sin \varphi \cos \vartheta \sin \left(\omega t+p_{i}\right) \cos \left(\omega t+p_{i}\right)\right]
\end{aligned}
$$

$$
\begin{aligned}
Q_{\varphi, L}= & q_{0} \omega R^{2} \sum_{i=1}^{N}\left[-B_{z} \sin ^{2} \vartheta \sin \left(\omega t+p_{i}\right) \cos \left(\omega t+p_{i}\right)\right. \\
& +B_{y} \sin \vartheta \cos \varphi \sin ^{2}\left(\omega t+p_{i}\right) \\
& +B_{y} \cos \vartheta \sin \vartheta \sin \varphi \sin \left(\omega t+p_{i}\right) \cos \left(\omega t+p_{i}\right) \\
& -B_{x} \sin \vartheta \sin \varphi \sin ^{2}\left(\omega t+p_{i}\right) \\
& \left.+B_{x} \cos \vartheta \sin \vartheta \cos \varphi \sin \left(\omega t+p_{i}\right) \cos \left(\omega t+p_{i}\right)\right] \\
& +q_{0} \vartheta R^{2} \sum_{i=1}^{N}\left[B_{z} \sin \vartheta \cos \vartheta \cos ^{2}\left(\omega t+p_{i}\right)\right. \\
& -B_{y} \cos { }^{2} \vartheta \sin \varphi \cos { }^{2}\left(\omega t+p_{i}\right) \\
& -B_{y} \cos \vartheta \cos \varphi \sin \left(\omega t+p_{i}\right) \cos \left(\omega t+p_{i}\right) \\
& -B_{x} \cos \vartheta \cos \varphi \cos ^{2}\left(\omega t+p_{i}\right) \\
& \left.+B_{x} \cos \vartheta \sin \varphi \sin \left(\omega t+p_{i}\right) \cos \left(\omega t+p_{i}\right)\right]
\end{aligned}
$$

where, as in the main text, we separated the terms proportional to $\omega$ from the others. On the other hand, the damping force yields

$$
\begin{aligned}
Q_{\vartheta, D}= & -k_{0} R^{2} \sum_{i=1}^{N}\left[\dot{\vartheta} \cos ^{2}\left(\omega t+p_{i}\right)\right. \\
& \left.+\dot{\varphi} \sin \vartheta \sin \left(\omega t+p_{i}\right) \cos \left(\omega t+p_{i}\right)\right] \\
Q_{\varphi, D}= & -k_{0} R^{2} \sum_{i=1}^{N}\left[\dot{\vartheta} \sin \vartheta \sin \left(\omega t+p_{i}\right) \cos \left(\omega t+p_{i}\right)\right. \\
& \left.+\dot{\varphi} \sin ^{2} \vartheta \sin ^{2}\left(\omega t+p_{i}\right)\right]
\end{aligned}
$$

We remember that here, $p_{i}=\frac{2 \pi}{N}(i-1)$ with $i=1, \ldots, N$. 
[1] A. Aharoni, Introduction to the Theory of Ferromagnetism (Oxford University Press, Oxford, 2000).

[2] G. Bertotti, I. Mayergoyz and C. Serpico, Nonlinear Magnetization Dynamic in Nanosystems (Elsevier, Oxford, 2000).

[3] M. Lakshmanan, The fascinating world of the Landau-Lifshitz-Gilbert equation: an overview, Phil. Trans. R. Soc. A 369, 1280 (2011).

[4] L. Landau and E. Lifshitz, On the theory of dispersion of magnetic permeability in ferromagnetic bodies, Phys. Zeitsch. der Sow. 8, 153 (1935).

[5] T. L. Gilbert, A Lagrangian formulation of the gyromagnetic equation of the magnetization field, Phys. Rev. 100, 1243 (1955) (abstract only); T. L. Gilbert, A Phenomenological Theory of Damping in Ferromagnetic Materials, IEEE Trans. Mag. 40, 3443 (2004).

[6] T. L. Gilbert and J. M. Kelly, Anomalous rotational damping in ferromagnetic sheets, Proceedings of the Pittsburgh Conference on Magnetism and Magnetic Materials, AIEE Special Publication T-78, 253 (1955).

[7] H. B. Callen, A ferromagnetic dynamical equation, J. Phys. Chem. Solids. 4, 256-270 (1958).

[8] J. C. Mallinson, On Damped Gyromagnetic Precession, IEEE Transaction on Magnetics 23, 2003 (1987).

[9] D. R. Fredkin and A. Ron, Microscopic derivation of the Landau-Lifschitz equation for ferromagnetic relaxation, Phys. Rev. B 61, 8654 (2000).

[10] G. Bertotti, I. D. Mayergoyz and C. Serpico, Identification of the damping coefficient in Landau-Lifshitz equation, Physica B 306, 102 (2001).

[11] S. Iida, The difference between Gilbert's and Landau-Lifshitz's equations, J. Phys. Chem. Solids. 24, 625 (1963).

[12] W. M. Saslow, Landau-Lifshitz or Gilber damping? That is the question, J. Appl. Phys. 105, 07D315 (2009).

[13] C. Chappert, A. Fert, and F. Nguyen Van Dau, The emergence of spin electronics in data storage, Nature Materials, 6, 813-823 (2007).

[14] S. Bandyopadhyay and M. Cahay, Electron spin for classical information processing: a brief survey of spin-based logic devices, gates and circuits, Nanotechnology 20, 412001 (2009).

[15] N. D'Souza, J. Atulasimha, and S. Bandyopadhyay, Four-state nanomagnetic logic using multiferroics, J. Phys. D: Appl. Phys. 44, 265001 (2011).

[16] N. A. Pertsev and H. Kohlstedt, Resistive switching via the converse magnetoelectric effect in ferromagnetic multilayers on ferroelectric substrates, Nanotechnology 21, 475202 (2010).

[17] C. W. Nan, M. I. Bichurin, S. Dong, D. Viehland, and G. Srinivasan, Multiferroic magnetoelectric composites: Historical perspective, status, and future directions, J. Appl. Phys. 103, 031101 (2008).

[18] S. Giordano, M. Goueygou, N. Tiercelin, A. Talbi, P. Pernod, and V. Preobrazhensky, Magneto-electro-elastic effective properties of multilayered artificial multiferroics with arbitrary lamination direction, Int. J. Eng. Sci. 78, 134 (2014).

[19] S.-T. Gu and Q.-C. He, Compact closed-form micromechanical expressions for the effective uncoupled and coupled linear properties of layered composites, Philosophical Magazine 95, 2793 (2015).

[20] S. Giordano, Explicit nonlinear homogenization for magnetoelectro-elastic laminated materials, Mech. Res. Comm. 55, 18 (2014).
[21] K. Roy, S. Bandyopadhyay, J. Atulasimha, Switching dynamics of a magnetostrictive single-domain nanomagnet subjected to stress, Phys. Rev. B 83, 224412 (2011).

[22] N. Tiercelin, Y. Dusch, A. Klimov, S. Giordano, V. Preobrazhensky, and P. Pernod, Room temperature magnetoelectric memory cell using stress-mediated magnetoelastic switching in nanostructured multilayers, Appl. Phys. Lett. 99, 192507 (2011).

[23] S. Giordano, Y. Dusch, N. Tiercelin, P. Pernod, and V. Preobrazhensky, Combined nanomechanical and nanomagnetic analysis of magnetoelectric memories, Phys. Rev. B 85, 155321 (2012).

[24] S. Giordano, Y. Dusch, N. Tiercelin, P. Pernod, and V. Preobrazhensky, Thermal effects in magnetoelectric memories with stress-mediated switching, J. Phys. D: Appl. Phys. 46, 325002 (2013).

[25] K. Roy, S. Bandyopadhyay, and J. Atulasimha, Energy dissipation and switching delay in stress-induced switching of multiferroic nanomagnets in the presence of thermal fluctuations, J. Appl. Phys. 112, 023914 (2012).

[26] W. F. Brown, Relaxational Behavior of Fine Magnetic Particles, J. Appl. Phys. 30, S130 (1959).

[27] W. F. Brown, Thermal Fluctuations of a Single-Domain Particle, J. Appl. Phys. 34, 1319 (1963).

[28] W. F. Brown, Thermal Fluctuations of a Single-Domain Particle, Phys. Rev. 130, 1677 (1963).

[29] W. F. Brown, Thermal fluctuation of fine ferromagnetic particles, IEEE Trans. Magn. 15, 1196 (1979).

[30] H. Risken, The Fokker-Planck equation (Springer-Verlag, Berlin, 1989).

[31] W.T. Coffey, Yu. P. Kalmykov, J.P. Waldron, The Langevin equation (World Scientific, Singapore, 2004).

[32] Y. P. Kalmykov and S. V. Titov, Matrix Elements of the System of Moment Equations Governing the Kinetics of Superparamagnetic Particles, Phys. Rev. Lett. 82, 2967 (1999).

[33] P.-M. Déjardin, D. S. F. Crothers, W. T. Coffey, and D. J. McCarthy, Interpolation formula between very low and intermediate-to-high damping Kramers escape rates for singledomain ferromagnetic particles, Phys. Rev. E 63, 021102 (2001).

[34] S. V. Titov, P.-M. Déjardin, H. El Mrabti, and Y. P. Kalmykov, Nonlinear magnetization relaxation of superparamagnetic nanoparticles in superimposed ac and dc magnetic bias fields, Phys. Rev. B 82, 100413(R) (2010).

[35] W. T. Coffey and Y. P. Kalmykov, Thermal fluctuations of magnetic nanoparticles: Fifty years after Brown, J. Appl. Phys. 112, 121301 (2012).

[36] S. Giordano, Y. Dusch, N. Tiercelin, P. Pernod, and V. Preobrazhensky, Stochastic magnetization dynamics in single domain particles, Eur. Phys. J. B 86, 249 (2013).

[37] N. L. Schryer, and L. R. Walker, The motion of $180^{\circ}$ domain wall in uniform dc magnetic fields, J. Appl. Phys. 45, 5406 (1974).

[38] S. S. P. Parkin, M. Hayashi, and L. Thomas, Magnetic DomainWall Racetrack Memory, Science 320, 190 (2008).

[39] J. H. Franken, H. J. M. Swagten, and B. Koopmans, Shift registers based on magnetic domain wall ratchets with perpendicular anisotropy, Nature Nanotechnology 7, 499 (2012).

[40] D. A. Allwood, G. Xiong, C. C. Faulkner, D. Atkinson, D. Petit, and R. P. Cowburn, Magnetic Domain-Wall Logic, Science 309, 1688 (2005). 
[41] T. Mathurin, S. Giordano, Y. Dusch, N. Tiercelin, P. Pernod and V. Preobrazhensky, Stress-mediated magnetoelectric control of ferromagnetic domain wall position in multiferroic heterostructures, Appl. Phys. Lett. 108, 082401 (2016).

[42] T. Mathurin, S. Giordano, Y. Dusch, N. Tiercelin, P. Pernod, and V. Preobrazhensky, Domain-wall dynamics in magnetoelastic nanostripes, Phys. Rev. B 95, 140405(R) (2017).

[43] M. D. Stiles, W. M. Saslow, M. J. Donahue, and A. Zangwill, Adiabatic domain wall motion and Landau-Lifshitz damping, Phys. Rev. B 75, 214423 (2007).

[44] N. Smith, Comment on "Adiabatic domain wall motion and Landau-Lifshitz damping", Phys. Rev. B 78, 216401 (2008).

[45] M.-C. Ciornei, J. M. Rubí, and J.-E. Wegrowe, Magnetization dynamics in the inertial regime: Nutation predicted at short time scales, Phys. Rev. B 83, 020410(R) (2011).

[46] M. Fähnle, D. Steiauf, and C. Illg, Generalized Gilbert equation including inertial damping: Derivation from an extended breathing Fermi surface model, Phys. Rev. B 84, 172403 (2011).

[47] J.-E. Wegrowe, and M.-C. Ciornei, Magnetization dynamics, gyromagnetic relation, and inertial effects, Am. J. Phys. 80, 607 (2012).

[48] E. Olive, Y. Lansac, and J.-E. Wegrowe, Beyond ferromagnetic resonance: The inertial regime of the magnetization, Appl. Phys. Lett. 100, 192407 (2012).

[49] E. Olive, Y. Lansac, M. Meyer, M. Hayoun, and J.-E. Wegrowe, Deviation from the Landau-Lifshitz-Gilbert equation in the inertial regime of the magnetization, J. Appl. Phys. 117, 213904 (2015).

[50] J.-E. Wegrowe and E. Olive, The magnetic monopole and the separation between fast and slow magnetic degrees of freedom, J. Phys.: Condens. Matter 28, 106001 (2016).

[51] R. Mondal, M. Berritta, and P. M. Oppeneer, Generalisation of Gilbert damping and magnetic inertia parameter as a series of higher-order relativistic terms, J. Phys.: Condens. Matter 30,
265801 (2018).

[52] T. Kikuchi and G. Tatara, Spin dynamics with inertia in metallic ferromagnets, Phys. Rev. B 92, 184410 (2015).

[53] Y. Li, V. V. Naletov, O. Klein, J. L. Prieto, M. Muñoz, V. Cros, P. Bortolotti, A. Anane, C. Serpico, and G. de Loubens, Nutation spectroscopy of a nanomagnet driven into deeply nonlinear ferromagnetic resonance, Phys. Rev. X 9, 041036 (2019).

[54] S. Ovcharenko, M. Gaponov, A. Klimov, N. Tiercelin, P. Pernod, E. Mishina, A. Sigov, and V. Preobrazhensky, Photoinduced spin dynamics in a uniaxial intermetallic heterostructure $\mathrm{TbCo}_{2} / \mathrm{FeCo}$, Scientific Reports 10, 15785 (2020).

[55] K. Neeraj, N. Awari, S. Kovalev, D. Polley, N. Z. Hagstrom, S. S. P. K. Arekapudi, A. Semisalova, K. Lenz, B. Green, J.C. Deinert, I. Ilyakov, M. Chen, M. Bowatna, V. Scalera, M. D’Aquino, C. Serpico, O. Hellwig, J.-E. Wegrowe, M. Gensch, and $\mathrm{S}$. Bonetti, Inertial spin dynamics in ferromagnets, Nat. Phys. (2020). https://doi.org/10.1038/s41567-020-01040-y

[56] E. Beaurepaire, J.-C. Merle, A. Daunois, and J.-Y. Bigot, Ultrafast Spin Dynamics in Ferromagnetic Nickel, Phys. Rev. Lett. 76, 4250 (1996).

[57] W. F. Brown, Micromagnetics (Interscience Publisher, New York, 1963).

[58] W. F. Brown, Magnetoelastic Interactions (Springer-Verlag, Berlin, 1966).

[59] L. D. Landau and E. M. Lifshitz, Electrodynamics of Continuous Media (Pergamon Press, London, 1984).

[60] J. A. Stratton, Electromagnetic theory (Mc Graw Hill, New York, 1941).

[61] H. Goldstein, C. Poole and J. Safko, Classical Mechanics (Addison Wesley, San Francisco, 2002).

[62] G. V. Skrotskii and L. V. Kurbatov, Theory of the anisotropy of the width of ferromagnetic resonance absorption line, Sov. Phys. JETP 8, 148 (1959).

[63] G. V. Skrotskii, The Landau-Lifshitz equation revisited, Sov. Phys. Usp. 27, 977 (1984).

[64] D. J. Griffiths, Dipoles at rest, Am. J. Phys. 60, 979 (1992). 